\title{
Gestión del Riesgo en la Implementación de Sistemas Fotovoltaicos en Proyectos de Extracción de Oro en Colombia a partir del Proceso de Análisis Jerárquico (AHP)
}

\author{
Cristian D. Ocampo ${ }^{2,3}$, Johnny Tamayo ${ }^{2,3}$ y Harold M. Castaño ${ }^{1,3}$ \\ (1) Facultad de Administración, Universidad Nacional de Colombia, Manizales, Colombia. \\ (e-mail: hmcastanoc@unal.edu.co) \\ (2) Facultad de Ingeniería y Arquitectura, Universidad Nacional de Colombia, Manizales, Colombia. \\ (e-mail: cdocampog@unal.edu.co; jatamayoar@unal.edu.co) \\ (3) Gestión del Conocimiento E-Business, Grupo de Investigación Académica.
}

Recibido Oct. 11, 2018; Aceptado Dic. 24, 2018; Versión final Ene. 21, 2019, Publicado Jun. 2019

\begin{abstract}
Resumen
Se identifican los riesgos que afectan la implementación de sistemas fotovoltaicos en proyectos de extracción de oro en Colombia y se prioriza la incidencia de estos riesgos en las fases del proyecto, mediante el método de decisión multicriterio Proceso de Análisis Jerárquico (AHP). Los riesgos identificados a partir de criterios técnicos fueron riesgo diseño, tecnología, y operación, mientras que a partir de criterios económicos fueron riesgo costos, externos y financieros. La fase de construcción es la etapa que probablemente se afecte más, debido a la aparición de estos riesgos, seguida por la fase de planeación y la fase de ejecución. De los criterios técnicos, el que tiene mayor peso es el riesgo diseño, seguido del riesgo operacional y del riesgo técnico. De los criterios económicos, el que tiene mayor peso es el riesgo costos, seguido de los riesgos financieros y de los riesgos externos.
\end{abstract}

Palabras clave: riesgos; AHP; PMI; fotovoltaico; gestión de proyectos; análisis jerárquico

\section{Risk Management in the Implementation of Photovoltaic Systems in Gold Extraction Projects in Colombia from the Hierarchy Analysis Process (AHP)}

\begin{abstract}
The risks that affect the implementation of photovoltaic systems in gold mining projects in Colombia are identified, and the incidence of these risks in the phases of the project is analyzed, using the multicriteria decision method Analysis Hierarchical Process (AHP). The risks identified from technical criteria were design, technology, and operational risk, while based on economic criteria, there were costs, external, and financial risks. The construction phase is the stage that is likely to be most affected, due to the appearance of these risks, followed by the planning phase and the execution phase. Of the technical criteria, the one with greater weight is the design risk, followed by operational risk and technical risk. Of the economic criteria, the one with greater weight is the cost risk, followed by financial risks and external risks.
\end{abstract}

Keywords: risks; AHP; PMI; photovoltaic; project management; hierarchy analysis 


\section{INTRODUCCIÓN}

La terminología asociada al riesgo en los proyectos y su concepción ha variado a través del tiempo, esto dado que el análisis de riesgo se ha venido realizando a través de la historia de manera no formal en innumerables situaciones. Riesgo ha estado siempre asociado a decisión, con algo que debe hacerse; con la ejecución de una acción que va dese lo trivial a lo muy importante (Cardona, 2001). Puede entenderse el riesgo como la probabilidad de que la amenaza se materialice, por lo que la gestión de los riesgos en proyectos energéticos tiene como finalidad evitar dichos fallos mejorando procesos, maximizando oportunidades e identificando y evitando amenazas futuras. A pesar de que existe una amplia variedad de sistemas metodológicos que gestionan el riesgo en un proyecto, aún no se ha llegado a un consenso sobre cuál metodología es la más efectiva, pues la mayoría dependen, en alguna medida, del punto de vista y de la experiencia de los interesados y gestores del proyecto.

La implementación de sistemas fotovoltaicos en proyectos mineros es una propuesta que ha empezado a tomar fuerza en países industrializados. Específicamente, en el contexto latinoamericano, Chile inicio a suplir la creciente demanda energética de sus operaciones incorporando energías no convencionales (energía solar, parques eólicos, entre otras). Por esta razón la gestión de riesgos en este tipo de proyectos, son estrategias necesarias para asegurar el suministro energético en el sector. Por ejemplo, el estudio de Parrado et al, (2015) señala que la implementación de energías renovables en las actividades mineras reduce costos de operación y son una opción viable que puede contribuir a la entrega continua y oportuna de electricidad sostenible. Por lo que proteger este tipo de activos de posibles amenazas presentes en el medio es un gran desafío. Para el caso que ocupa esta investigación se acudió al uso de técnicas de investigación las cuales se emplearon para estructurar un diseño metodológico de tipo descriptivo, apoyado en métodos cualitativos y cuantitativos (mixtos), usando instrumentos de recolección de información tales como cuestionarios, entrevistas, documentos (Hernández et al., 2014). Con el desarrollo de esta metodología se pretende contrastar a partir de un modelo teórico elementos de juicio que sirvan en la identificación, ponderación y priorización de los riesgos. Finalmente, este estudio permite brindar herramientas prácticas que permitan diagnosticar riesgos apoyados en métodos de decisión multicriterio-AHP.

Para el desarrollo de esta investigación, se tuvieron en cuenta los planteamientos de Saaty (1987), en relación con la técnica de Análisis Procesos Jerárquicos (AHP). Por consiguiente, este documento da indicaciones de cómo se puede tratar el riesgo y la incertidumbre utilizando la técnica AHP a partir del enfoque de medición por escalas de relación. Prasanta (2001) brinda un enfoque cuantitativo para la gestión del riesgo en la construcción de un oleoducto, a partir de la técnica del AHP y del análisis de árboles de decisión. El autor identificó los factores de riesgos, cuantificó sus efectos mediante la determinación de probabilidad y del impacto, y generó respuestas alternativas con una implicación de costos para mitigar los riesgos cuantificados. Otra investigación que se tomó como referencia de estudio fue la realizada por Guerrero-Liquet, et al., (2016), en donde apoyados en investigaciones relacionadas con métodos de toma de decisión multicriterio, en la gestión de proyectos, analizan riesgos de tipo técnicos y financieros e identifican los riesgos con probabilidad de ocurrencia que afectan la inversión en cualquier tipo de instalación de energía renovable.

\section{METODOLOGÍA}

El diseño metodológico propuesto para esta investigación se caracteriza por ser de tipo descriptivo, utiliza un método de investigación mixto (cualitativo - cuantitativo), las fuentes y técnicas de recolección de información utilizadas en la investigación fueron las siguientes: Primarias (se apoyó en técnicas de recolección de información tales como lluvia de ideas, cuestionarios y entrevistas semi estructuradas, donde se realizaron preguntas abiertas a los expertos) y secundarias (se acudió a la revisión estructurada de documentos relacionados a proyectos de carácter energético, artículos académicos, libros, tesis, páginas web, textos, artículos de prensa entre otros.)

A partir de técnicas de evaluaciones cualitativas y cuantitativas se hace una ponderación y priorización de los riesgos en el proyecto, para lograr este objetivo es frecuente el uso de técnicas y técnicas que se apoyan en la experiencia y en el juicio subjetivo del investigador. En el desarrollo de esta investigación, se utilizó la técnica llamada Proceso de Análisis Jerárquico (AHP), método propuesto por el profesor Thomas Saaty de la Universidad de Pittsburg, a finales de la década de los 70. La aplicación del Proceso de Análisis Jerárquico (AHP), se hace con la finalidad de ponderar y priorizar los riesgos identificados a través de una estructura jerárquica. Una vez identificados los atributos y los sub-atributos, se determina la importancia relativa que tiene cada uno dentro de cada categoría. Además, usando una escala de prioridades se compara la importancia relativa de un riesgo con respecto a otro, lo cual se apoya en la experiencia y en el juicio subjetivo del investigador y de esta manera, se sintetizan los juicios emitido, proporcionado un ranking de alternativas de acuerdo con los pesos obtenidos. La metodología AHP propone una forma de clasificar el pensamiento analítico, incluyendo tres principios básicos: el principio de construir jerarquías, el principio de prioridad y el principio de consistencia lógica (Guerrero-Liquet et al., 2015). 
Esta metodología toma especial relevancia, dado que evalúa aspectos intangibles como el riesgo y ayuda a priorizar de manera sistemática los riesgos que pueden llegar a afectar un proyecto. La elección de la metodología AHP se debió a que presenta un soporte matemático valido; el análisis de los problemas se hace a partir de la descomposición y el desglose de los mismos; mide criterios cuantitativos y cualitativos mediante una escala común; a partir de índices de inconsistencias s e pueden verificar errores en el desarrollo de las evaluaciones, lo que facilita hacer las correcciones que sean del caso; es de fácil uso; permite que personas o grupos de interés participen; y los resultados se pueden complementar con otros métodos matemáticos de optimización (Toskano, 2005).

\section{IDENTIFICACIÓN DE LOS RIESGOS}

La implementación metodológica para la identificación de los riesgos pretende combinar diferentes herramientas que ayuden a identificar, ponderar y priorizar los riesgos con base en la afectación de las fases del proyecto. Mediante reuniones concertadas con expertos, y con soporte en técnicas como la lluvia de ideas, el análisis de supuestos, la revisión documental y la entrevista no estructurada, se lograron identificar las oportunidades, las debilidades, las fortalezas y las amenazas que involucra el proyecto.

En adición, se construyó una matriz DOFA (debilidades, oportunidades, fortalezas y amenazas) con la finalidad de identificar los problemas que pueden afectar el desempeño y la rentabilidad de implementar un sistema fotovoltaico en proyectos de extracción de oro en Colombia. Este análisis arrojó información concerniente a temas relacionado con costos de inversión, normatividad actual de incentivos de uso de energías renovables, volatilidad precio energía, eficiencia, ahorro del sistema fotovoltaicos, entre otros. Finalmente, con esta información, se obtuvieron estrategias ofensivas (fortalezas-oportunidades), de reorientación (fortalezas-amenazas), defensivas (debilidades-Oportunidades) y de supervivencia (AmenazasDebilidades) (Rojas, 1999)

La identificación y la categorización de los riesgos se logró mediante la realización de reuniones con expertos de diferentes disciplinas (Geólogo especialista en gerencia de proyectos mineros; ingeniero industrial con doctorado en ingeniera, industria y organizaciones; ingeniero electrónico con maestría en automatización industrial; ingeniero eléctrico con doctorado en ingeniería eléctrica; ingeniero de sistemas especialista en gestión de proyectos PMI;), los cuales tienen amplia experiencia en temas relacionados con la exploración y explotación de recursos energéticos no renovables, en planificación y evaluación de proyectos PMI, desarrollo y aplicación de proyectos energéticos enfocados al uso de energías renovables, quienes, a partir de lluvia de ideas, entrevistas no estructuradas, resolución de cuestionarios y aplicación de técnicas de diagramación, lograron caracterizar riesgos asociados con este tipo de proyectos, los cuales, a partir de lluvia de ideas, entrevistas no estructuradas, resolución de cuestionarios y aplicación de técnicas de diagramación, lograron caracterizar riesgos asociados con este tipo de proyectos. Luego de la identificación de estos riesgos se desarrollaron estrategias que ayudaron a determinar el origen de éstos, y se agruparon en paquetes de trabajo para hacer más sencillo su análisis.

Los riesgos identificados en la investigación (figura 1) fueron los siguientes: a partir de criterios técnicos, se identificaron riesgo diseño, riesgo tecnológico y riesgo operacional; a partir de criterios económicos, se identificaron riesgo costos, riesgos financieros y riesgos externos.

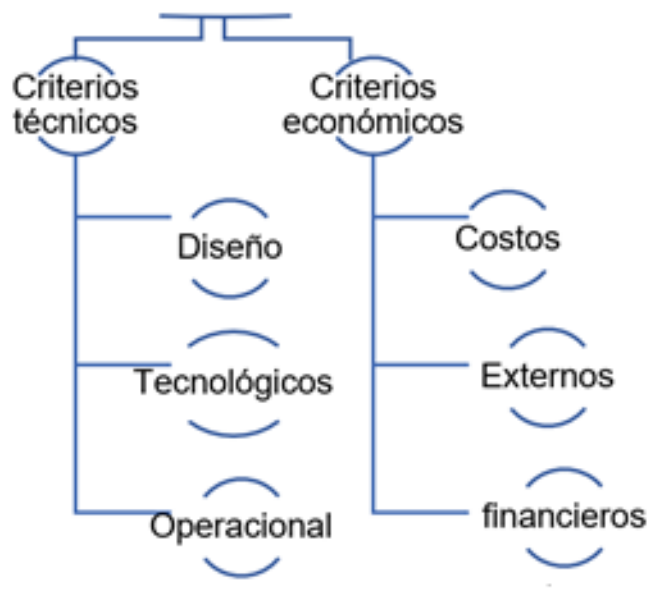

Fig. 1: Riesgos identificados en el proyecto 


\section{MODELO PARA LA EVALUACIÓN DE LOS RIESGOS}

Para el desarrollo de este proyecto se decidió usar el software Superdecision $®$, el cual se utiliza para resolver problemas de decisión multicriterio, en este caso, procesos de análisis jerárquicos (figura 2). El principio matemático de este programa se sustenta en: la teoría de matrices, la teoría de grafos, la teoría de decisiones colectivas, y la teoría de las organizaciones. Cabe agregar que la información e interpretación que arroja el software dependerá de las preferencias del decisor y los objetivos previamente establecidos. Es de considerar que el decisor que utiliza el software debe estar familiarizado con las alternativas que va a valorar, lo que señala que es necesario familiarizado con las estructuras de modelo posibles para así, diseñar su propio modelo de evaluación. El decisor debe tener el conocimiento suficiente para valorar todas las alternativas, lo que va a depender de los criterios que él mismo establezca. Por último, los resultados que arroja el programa dependerán de la fórmula de síntesis utilizada para elegir preferencias entre los diferentes niveles o jerarquías del modelo empleado, dado que el programa no ofrece cuál es el modelo o la fórmula más conveniente, lo que dependerá del juicio del decisor (PRONACOSE, 2014).

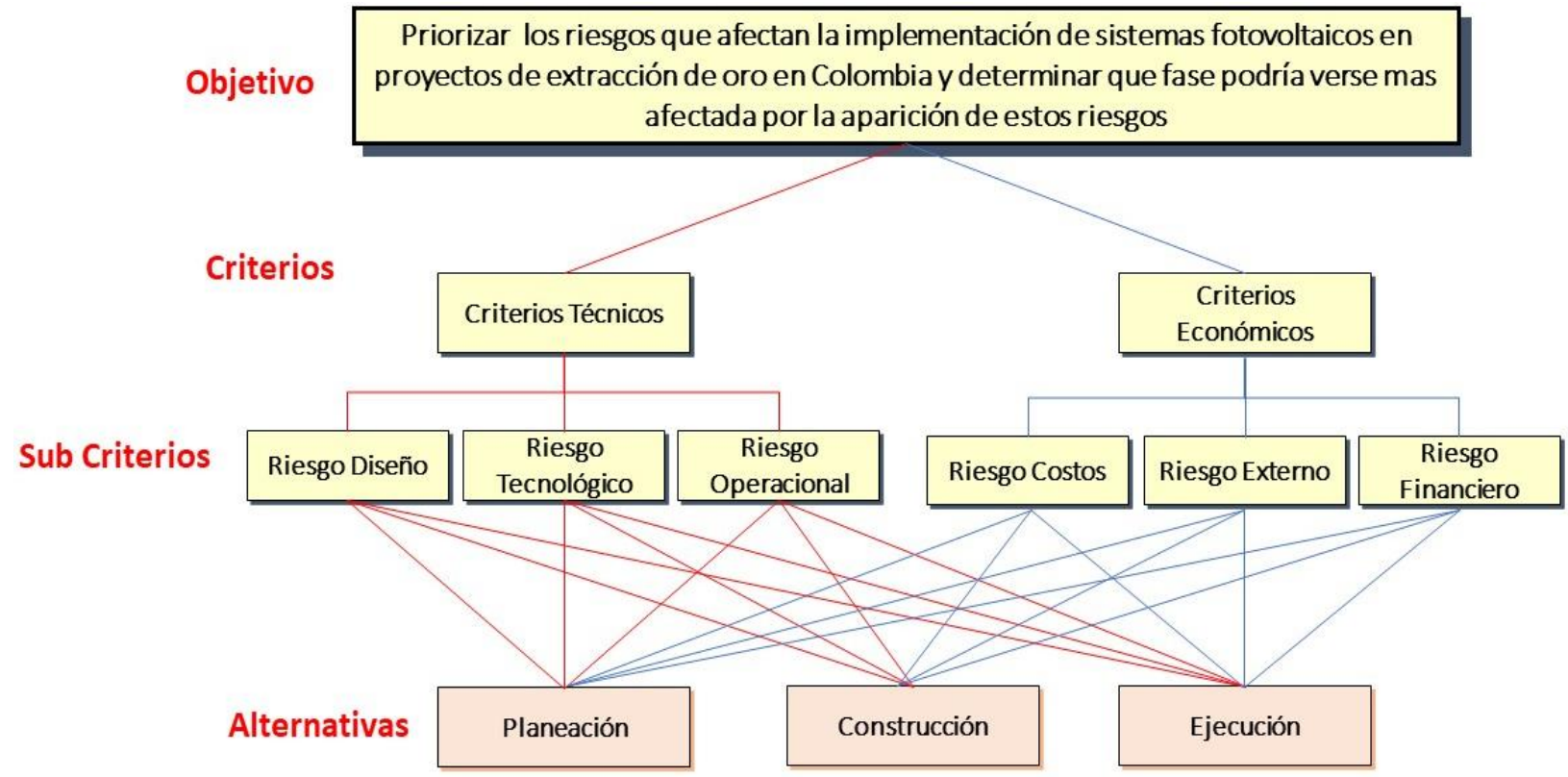

Fig. 2: Jerarquización problema de estudio.

Jerarquizado el problema se procede a desarrollar un proceso de decisiones mediante evaluaciones subjetivas. Se compara entonces la importancia relativa de los criterios analizados ( $¿ E s$ el criterio1 igual o mas importante que el criterio 2?) y se especifica su preferencia respecto a las alternativas presentadas. (Con relación al criterio 1: ¿Es la alternativa 1 igual o mas importante que la alternativa 2?) de acuerdo con la calificación o escala de Satty (tabla 1). Allí se establecen prioridades y se emiten juicios.

Tabla 1: Escala de Satty (Adaptado de Saaty, T. L., \& Vargas, L. G., 2012)

\begin{tabular}{|c|l|l|}
\hline Valor & Escala verbal $(A H P)$ & Comentarios \\
\hline 1 & Igual importancia & A y B tienen la misma importancia \\
\hline 3 & Importancia moderada & A es ligeramente más importante que B \\
\hline 5 & Importancia fuerte & A es más importante que B \\
\hline 7 & Importancia muy fuerte & A es mucho más importante que B \\
\hline 9 & Extrema importancia & A es extremadamente más importante que B \\
\hline $2,4,6,8$ & Valores intermedios & Valores intermedios \\
\hline
\end{tabular}

Se muestran a continuación las comparaciones realizadas por los expertos, estas fueron desarrolladas utilizando el software Superdecision ${ }^{\circledR}$, se compara la importancia relativa entre los criterios técnicos y económicos. (figura 3). Luego se determina qué riesgo es más relevante en función de los criterio técnicos y económicos, como se muestra en las figuras 4 y 5 . Después se procede a comparar las fases del proyecto en función de cada uno de los sub-criterio técnicos y así determinar que fases del proyecto tiene más afectación de acuerdo con el sub-criterio evaluado. (figuras 6,7 y 8). Finalmente, se procede a comparar las fases del proyecto en función de cada uno de los sub-criterios económicos y así determinar que fases del proyecto tiene más afectación de acuerdo con el sub-criterio evaluado. (Figuras 9, 10 y 11) 
Graphical Verbal Matrix Questionnaire Direct

Comparisons wrt "OBJETIVO" node in "CRITERIOS" cluster

R1.TECNICOS is equally as impacta as R2.ECONOMICOS

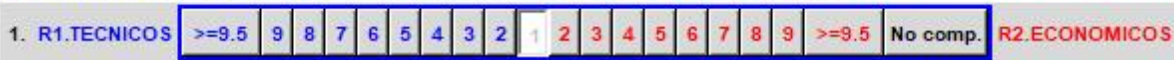

\begin{tabular}{|c|c|}
\hline Normal - & Hybrid - \\
\hline \multicolumn{2}{|c|}{ Inconsistency: 0.00000} \\
\hline R1.TECNIC & 0.50000 \\
\hline R2.ECONOM & 0.50000 \\
\hline
\end{tabular}

Fig.3: Matriz de comparación criterio técnico vs riesgo económico (Datos de Superdecision $\circledast$, 2018)

Graphical Verbal Matrix Questionnaire Direct

Comparisons wit "R1.TECNICOS" node in "CRITERIOS" cluster

R1.1_DISEÑO is strongly more impacta than R1.2_TECNOLOGICO

\begin{tabular}{|c|c|c|c|c|c|c|c|c|c|c|c|c|c|c|c|c|c|c|c|c|c|}
\hline 1. & R1.1_DISEÑO & $>=9.5$ & 9 & 8 & 7 & 6 & 5 & 4 & 3 & 2 & 2 & 3 & 4 & 5 & 6 & 7 & 8 & 9 & $>=9.5$ & No comp. & R1.2_TECNOLOGIC \\
\hline & R1.1_DISEÑO & $>=9.5$ & 9 & 8 & 7 & 6 & 5 & 4 & 3 & 2 & 2 & 3 & 4 & 5 & 6 & 7 & 8 & 9 & $>=9.5$ & No comp. & R1.3_OP \\
\hline & $\mathrm{CI}$ & $>=9.5$ & 9 & 8 & & 6 & 5 & 4 & 3 & 2 & 2 & & 4 & 5 & 6 & 7 & 8 & 9 & $>=9.5$ & No comp. & R1.3_OPERA \\
\hline
\end{tabular}

\begin{tabular}{|l|l|} 
Normal -1 & Hybrid - \\
Inconsistency: 0.03703 \\
\begin{tabular}{|l|l|}
\hline R1.1_DISE & 0.63699 \\
\hline R1.2_TECN & 0.10473 \\
\hline R1.3_OPER & 0.25829 \\
\hline
\end{tabular}
\end{tabular}

Fig.4: Matriz comparación sub-criterios técnicos en función de criterio técnico (Datos de Superdecision ${ }^{\circledR}, 2018$ )

Graphical Verbal Matrix Questionnaire Direct

Comparisons wrt "R2.ECONOMICOS" node in "CRITERIOS" cluster

R2.1_COSTOS is strongly more impacta than R2.2_EXTERNOS

1. R2.1_costos \begin{tabular}{|l|l|l|l|l|l|l|l|l|l|l|l|l|l|l|l|l|l|l|l|}
\hline$>9.5$ & 9 & 8 & 7 & 6 & 5 & 4 & 3 & 2 & & 2 & 3 & 4 & 5 & 6 & 7 & 8 & 9 & $>=9.5$ & No comp. R2.2_EXTERNOS \\
\hline
\end{tabular}

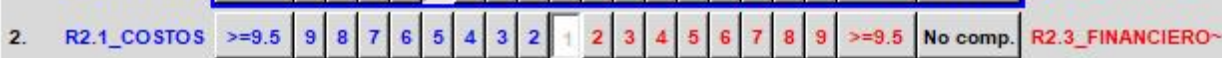

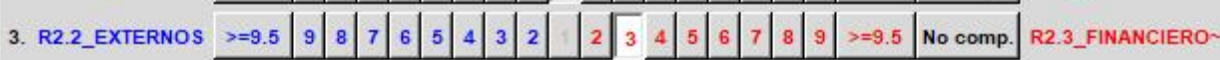

\begin{tabular}{|l|l|}
\hline Normal - & \multicolumn{1}{c|}{ Hybrid -} \\
Inconsistency: 0.02795 \\
\hline R2.1_COST & 0.48064 \\
\hline R2.2_EXTE & 0.11397 \\
\hline R2.3_FINA & 0.40539 \\
\hline
\end{tabular}

Fig.5: Matriz comparación sub-criterios económicos en función de criterio económico (Datos de Superdecision $\AA^{8}, 2018$ )

Graphical Verbal Matrix Questionnaire Direct

Comparisons wit "R1.1_DISEÑO" node in "ALTERNATIVES" cluster

F1.PLANEACIÓN is strongly more impacta than F2. CONSTRUCCION

\begin{tabular}{lll|l|l|l|l|l|l|l|l|l|l|l|l|l|l|l|l|l|l|l|} 
1. F1.PLANEACIÓN & $>=9.5$ & 9 & 8 & 7 & 6 & 5 & 4 & 3 & 2 & & 2 & 3 & 4 & 5 & 6 & 7 & 8 & 9 & $>=9.5$ & No comp. & F2.CONSTRUCCION \\
2. F1.PLANEACIÓN & $>=9.5$ & 9 & 8 & 7 & 6 & 5 & 4 & 3 & 2 & & 2 & 3 & 4 & 5 & 6 & 7 & 8 & 9 & $>=9.5$ & No comp. F3.EJECUCIÓN \\
3. F2.CONSTRUCCION & $>=9.5$ & 9 & 8 & 7 & 6 & 5 & 4 & 3 & 2 & & 2 & 3 & 4 & 5 & 6 & 7 & 8 & 9 & $>=9.5$ & No comp. F3.EJECUCIÓN
\end{tabular}

\begin{tabular}{|l|l|}
\hline Normal - & Hybrid -1 \\
Inconsistency: & 0.06239 \\
\hline F1.PLANEA & 0.73064 \\
\hline F2.CONSTR & 0.18839 \\
\hline F3.EJECUC & 0.08096 \\
\hline
\end{tabular}

Fig.6: Matriz comparación fases del proyecto (alternativas) en función de sub-criterio riesgo diseño (Datos de Superdecision $\AA$, 2018)

Graphical Verbal Matrix Questionnaire Direct

Comparisons wit "R1.2_TECNOLOGICO" node in "ALTERNATIVES" cluster

F2.CONSTRUCCION is moderately more impacta than F1.PLANEACIÓN

1. F1.PLANEACIÓN \begin{tabular}{|l|l|l|l|l|l|l|l|l|l|l|l|l|l|l|l|l|l|l|l|}
$>=9.5$ & 9 & 8 & 7 & 6 & 5 & 4 & 3 & 2 & & 2 & 3 & 4 & 5 & 6 & 7 & 8 & 9 & $>=9.5$ & No comp. F2.CONSTRUCCION
\end{tabular}

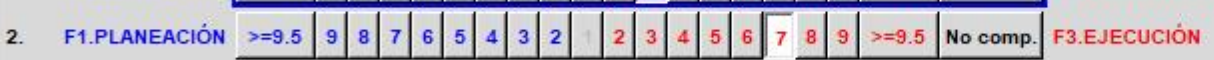

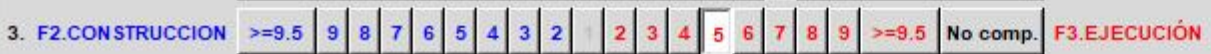

\begin{tabular}{|c|c|}
\hline Normal $=$ & Hybrid - \\
\hline \multicolumn{2}{|c|}{ Inconsistency: 0.06239} \\
\hline F1.PLANEA & 0.08096 \\
\hline F2.CONSTR & 0.18839 \\
\hline F3.EJECUC & 0.73064 \\
\hline
\end{tabular}

Fig.7: Matriz comparación fases del proyecto (alternativas) en función de sub-criterio riesgo tecnológico (Datos de Superdecision ${ }^{\circledR}, 2018$ )

Graphical Verbal Matrix Questionnaire Direct

Comparisons wit "R1.3 OPERACIONALES" node in "ALTERNATIVES" cluster

F2. CONSTRUCCION is very strongly more impacta than F1.PLANEACIÓN

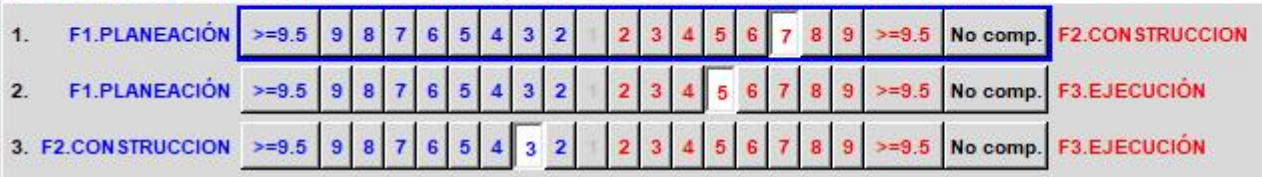

\begin{tabular}{|c|c|}
\hline Normal $\longrightarrow$ & Hybrid - \\
\hline \multicolumn{2}{|c|}{ Inconsistency: 0.06239} \\
\hline F1.PLANE & 0.0719 \\
\hline F2.CON & 0.6 \\
\hline$=31$ & 0. \\
\hline
\end{tabular}

Fig.8: Matriz comparación fases del proyecto (alternativas) en función de sub-criterio riesgo operacional (Datos de Superdecision $\left.\AA^{\circledR}, 2018\right)$ 
Graphical Verbal Matrix Questionnaire Direct

Comparisons wit "R2.1_COSTOS" node in "ALTERNATIVES" cluster

F2. CONSTRUCCION is very strongly more impacta than F1.PLANEACIÓN

1. F1.PLANEACIÓN \begin{tabular}{r|c|c|c|c|c|c|c|c|c|c|c|c|c|c|c|c|c|c|c|}
$>=9.5$ & 9 & 8 & 7 & 6 & 5 & 4 & 3 & 2 & & 2 & 3 & 4 & 5 & 6 & 7 & 8 & 9 & $>=9.5$ & No comp. F2.CON STRUCCION
\end{tabular}

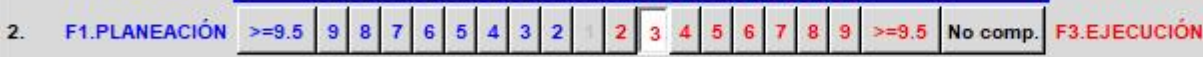

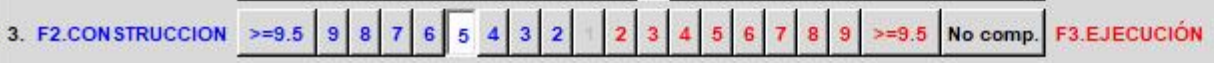

\begin{tabular}{|l|l|}
\hline Normal - & Hybrid -1 \\
Inconsistency: & 0.06239 \\
\hline F1.PLANEA & 0.08096 \\
\hline F2.CONSTR & 0.73064 \\
\hline F3.EJECUC & 0.18839 \\
\hline
\end{tabular}

Fig.9: Matriz comparación fases del proyecto (alternativas) en función de subcriterio riesgo costos (Datos tomados de Superdecision $\left.\AA^{\circledR}, 2018\right)$

Graphical Verbal Matrix Questionnaire Direct

Comparisons wit "R2.2_EXTERNOS" node in "ALTERNATIVES" cluster

F2.CONSTRUCCION is moderately more impacta than F1.PLANEACIÓN

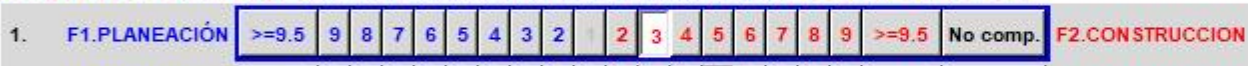

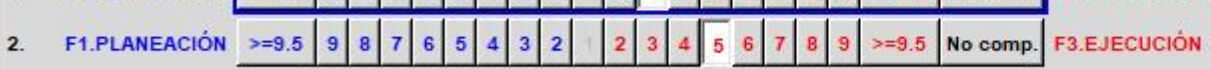

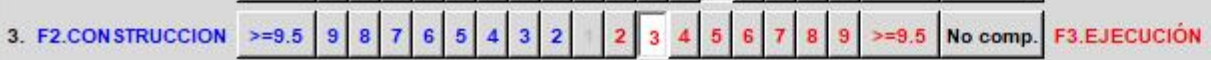

\begin{tabular}{|c|c|}
\hline Normal - & Hybrid - \\
\hline \multicolumn{2}{|c|}{ Inconsistency: 0.03703} \\
\hline F1.PLANEA & 0.10473 \\
\hline F2.CONSTR & 0.25828 \\
\hline F3.EJECUC & 0.63699 \\
\hline
\end{tabular}

Fig.10: Matriz comparación fases del proyecto (alternativas) en función de sub-criterio riesgo externos (Datos tomados de Superdecision $\AA^{\circledR}, 2018$ )

Graphical Verbal Matrix Questionnaire Direct

Comparisons wit "R2.2 EXTERNOS" node in "ALTERNATIVES" cluster

F2. CONSTRUCCION is moderately more impacta than F1.PLANEACIÓN

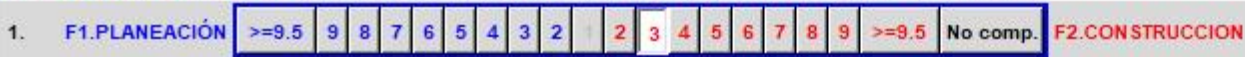

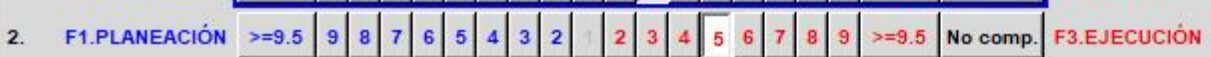

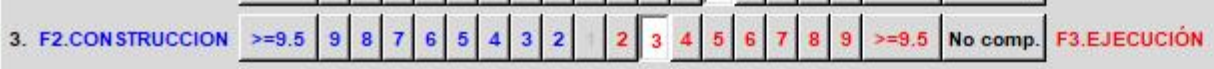

\begin{tabular}{|l|l|}
\hline Normal -1 & Hybrid -1 \\
Inconsistency: 0.03703 \\
\hline F1.PLANEA & 0.10473 \\
\hline F2.CONSTR & 0.25828 \\
\hline F3.EJECUC & 0.63699 \\
\hline
\end{tabular}

Fig.11: Matriz comparación fases del proyecto (alternativas) en función de sub-criterio riesgo financieros (Datos tomados de Superdecision $\AA$, 2018)

Finalizada la fase de comparaciones, se procede a realizar el análisis y la normalización de los resultados. Uno de los atributos del software es la normalización automática de la información obtenida, es decir, transforma una variable aleatoria (vectores de criterios y vectores de alternativas), la cual, tiene alguna distribución, en una nueva variable aleatoria con distribución normal o aproximadamente normal. De esta forma, es posible obtener más información y de manera precisa para la evaluación del proyecto.

El análisis siguiente (tabla 2) muestra los pesos locales y pesos globales de las comparaciones realizadas. Según estos resultados, para el criterio técnico $(0,25)$, el sub-criterio con mayor peso es el riesgo diseño $(0,15925)$, luego se tienen los riesgos operacionales $(0,06457)$, y, finalmente, se tiene el riesgo tecnológico $(0,02618)$. De otro lado, tenemos que, para el criterio económico $(0,25)$, el subcriterio riesgo costos $(0,12016)$ tiene mayor peso, seguido de riesgos financieros $(0,10135)$ y de riesgos externos $(0,02849)$. Con relación a las fases del proyecto, tenemos que la fase construcción $(0,441)$ es la que probablemente sea más afectada por la aparición de riesgos asociados a criterios técnicos y económicos, seguido por la fase de planeación $(0,294)$ y fase ejecución $(0,263)$.

Tabla 2: Pesos locales y pesos globales del proyect

\begin{tabular}{|l|l|l|}
\hline Factores & Pesos Locales & Pesos globales \\
\hline Criterio Técnicos & 0,25 & 0,166667 \\
\hline Riesgo Diseño & 0,15952 & 0,106164 \\
\hline Riesgo Tecnológico & 0,02618 & 0,017455 \\
\hline Riesgo Operacional & 0,06457 & 0,043048 \\
\hline Criterio Económicos & 0,25 & 0,166667 \\
\hline Riesgo Costos & 0,12016 & 0,080107 \\
\hline Riesgos Externos & 0,02849 & 0,018995 \\
\hline Riesgos Financieros & 0,10135 & 0,067565 \\
\hline Fase Planeación & 0,29476 & 0,098523 \\
\hline Fase Construcción & 0,44143 & 0,147142 \\
\hline Fase Ejecución & 0,26381 & 0,087929 \\
\hline \multicolumn{2}{|c}{}
\end{tabular}


Los riesgos analizados en el proyecto (figura 12) se grafican en función de sus pesos locales y pesos globales. Estos valores fueron determinados a partir de las comparaciones previamente realizadas. Para un análisis más exhaustivo, el software permite crear modelos, mostrando posibles alternativas o los resultados según cambio (hipotético) en los criterios de evaluación a partir de un análisis de sensibilidad, para este caso, se podrá determinar qué fase del proyecto se verá más afectada a medida que se le da mayor o menor peso a un criterio en específico (técnico u económico). Por lo tanto, a partir de la creación de escenarios, se pueden analizar detenidamente sus resultados.

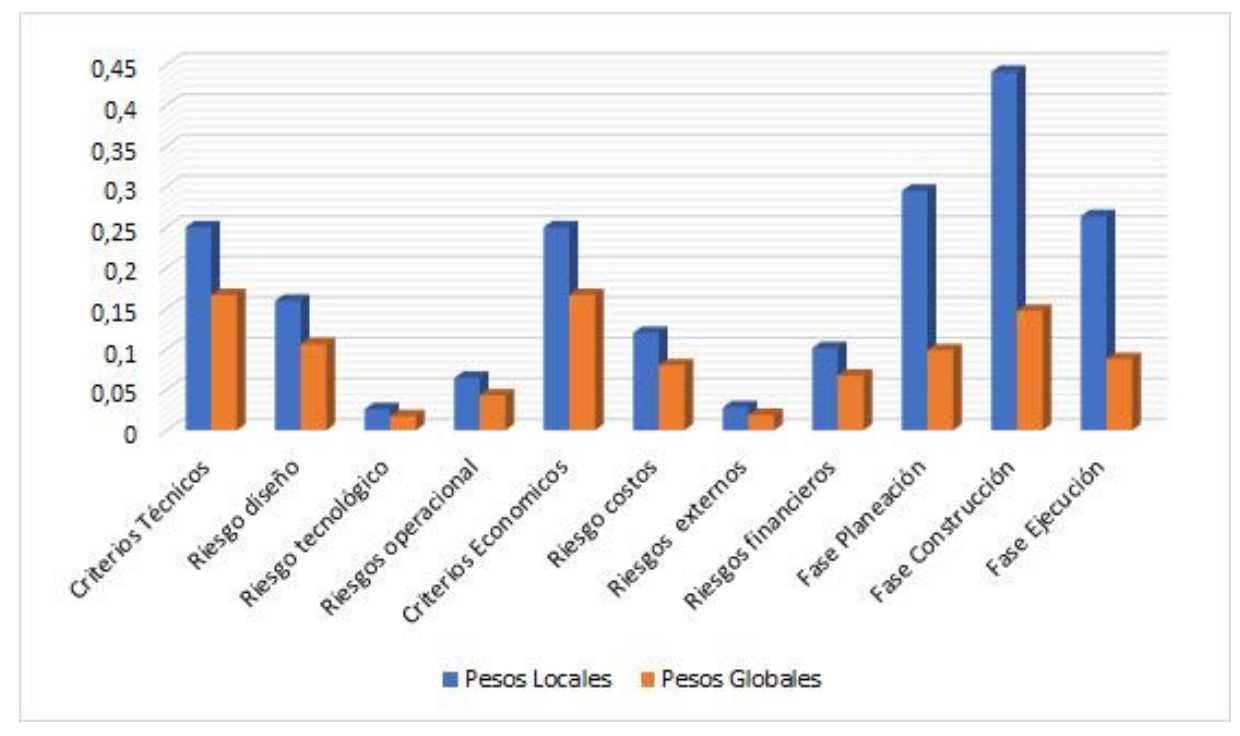

Fig. 12: Distribución pesos del proyecto

El análisis de sensibilidad llevado a cabo (figura 13) se muestra en función del criterio técnico; los pesos de los criterios se trazan en el eje $\mathrm{X}$ y los pesos de las alternativas (fases) se trazan en el eje $\mathrm{Y}$. Así, al asignarle una mayor magnitud o peso al criterio técnico $(0,8)$, se observa que los pesos en las fases cambian: planeación $(0,412)$, construcción $(0,362)$ y ejecución $(0,226)$. Este análisis muestra que los riesgos asociados con el criterio técnico podrían afectar en mayor medida las fases planeación y construcción. El razonamiento es lógico pues en estas fases se lleva a cabo el pre-diseño, diseño y construcción del proyecto, y, por la naturaleza del riesgo, es necesario desarrollar un plan de respuesta a los riesgos eficiente, buscando evitar que los riesgos de carácter técnico lleguen a dejar inviable el proyecto.

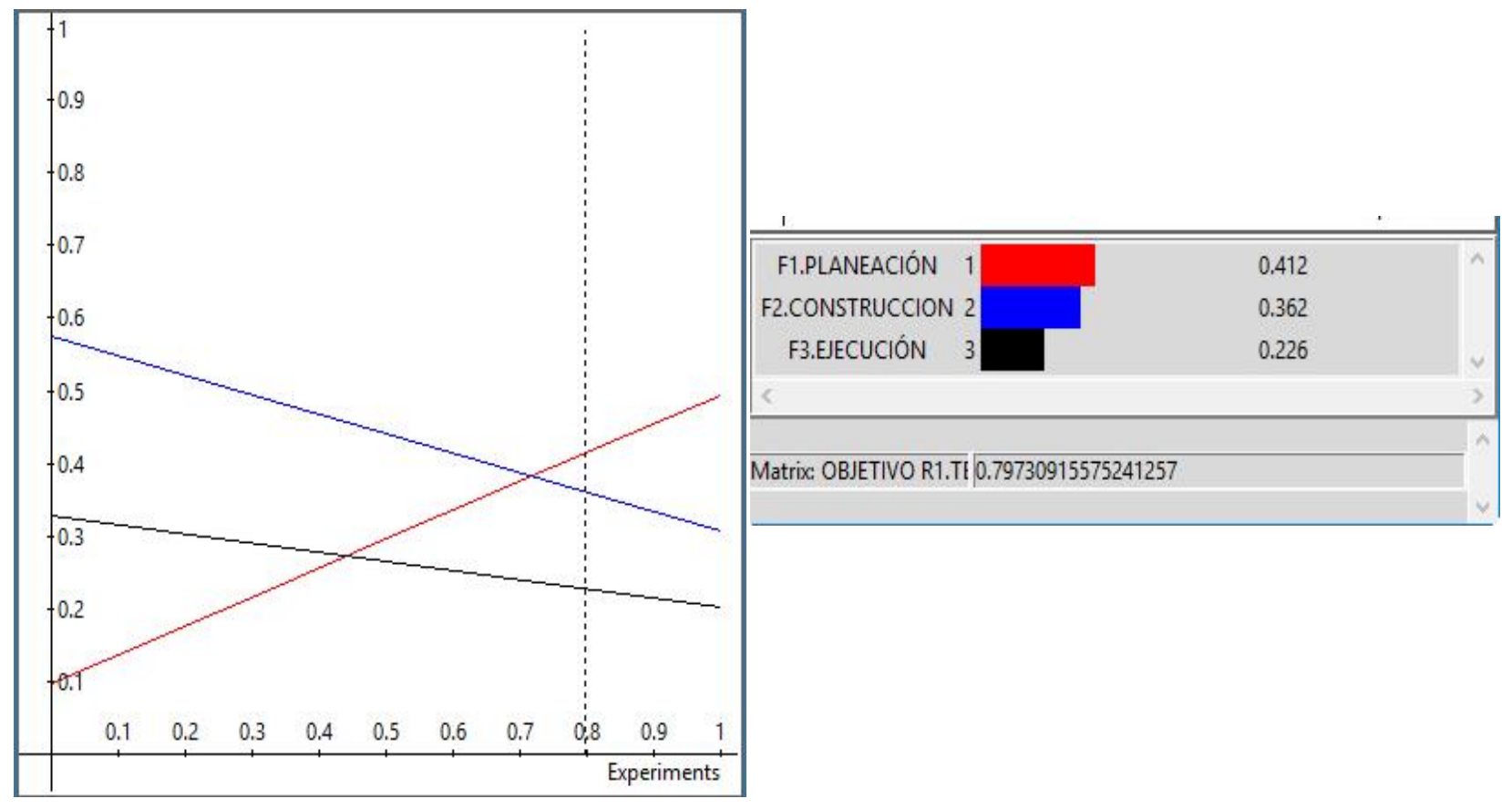

Fig. 13: Análisis de sensibilidad en función del criterio técnico. (Datos tomados de Superdecision $\circledast$, 2018) 
El siguiente análisis de sensibilidad (figura 14) se realiza en función del criterio económico. Como en el caso anterior, al asignarle una magnitud mayor a este criterio $(0,7)$ se observa que la fase que tiene una posibilidad mayor de ser afectada por este incremento es la construcción $(0,495)$, seguida por la fase ejecución $(0,289)$ y, por último, la fase planeación $(0,216)$. Riesgos asociados con los altos costos de inversión, recuperación de la inversión, problemas de liquidez, entre otros, tienden a golpear las fases de construcción y ejecución de los proyectos.

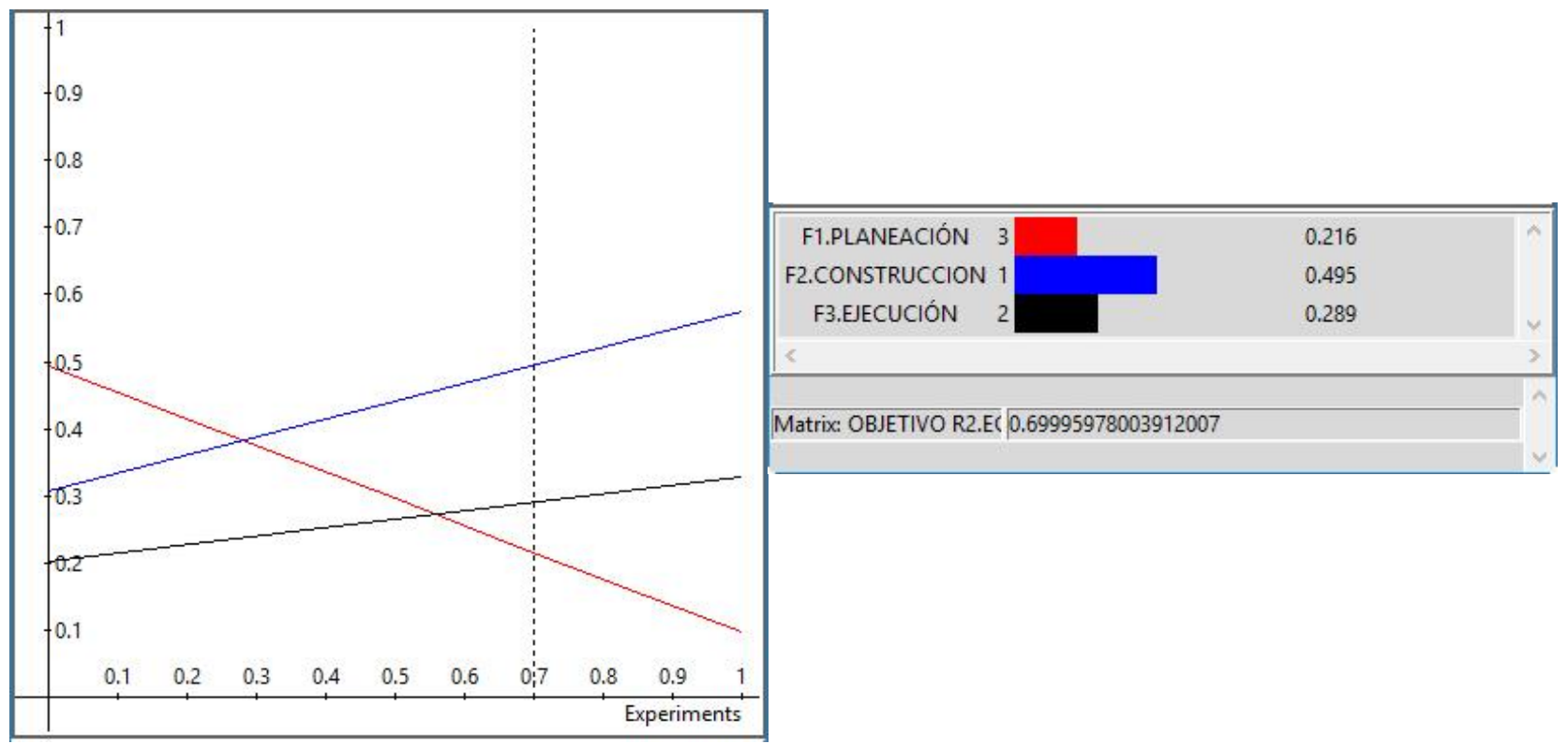

Fig.14: Análisis de sensibilidad en función del criterio económico. (Datos tomados de Superdecision $\AA_{\text {, 2018) }}$

\section{PLAN DE MANEJO DE LOS RIESGOS}

Realizar una planificación de respuesta hacia los riesgos, que sea eficiente, implica que es necesario definir planes de acción que ayuden a dar respuestas a los riesgos y a definir qué objetivos pueden verse afectados. A partir de las evaluaciones realizadas se logró a identificar riesgos y se desarrolló planes de respuesta. En la tabla 3 se muestra la matriz de administración de riesgos generada para este estudio.

Tabla 3: Matriz de administración de criterios técnicos y económicos

\begin{tabular}{|c|c|c|}
\hline Criterios & Respuestas & Objetivo afectado \\
\hline Riesgo diseño & $\begin{array}{l}\text { Realizar reuniones periódicas para analizar el desempeño del } \\
\text { sistema y el entorno del proyecto para la toma de decisiones. } \\
\text { Revisión diseño de las instalaciones } \\
\text { Revisión de requerimientos, especificaciones, ingeniería }\end{array}$ & $\begin{array}{l}\text { Gestión Alcance } \\
\text { Gestión Tiempo }\end{array}$ \\
\hline Riesgos operacionales & $\begin{array}{l}\text { Programas de Auditoria } \\
\text { Relaciones publicas } \\
\text { Realizar reuniones capacitaciones } \\
\text { Aseguramiento de la calidad, administración y estándares } \\
\text { Las reservas de gestión del proyecto } \\
\text { Rediseñar cronogramas, mejorar recursos tanto de personal como de } \\
\text { equipo. } \\
\text { Tener un grupo multidisciplinario de trabajo con conocimiento de las } \\
\text { reglamentaciones técnicas exigidas para el tipo de proyecto a } \\
\text { ejecutar }\end{array}$ & $\begin{array}{l}\text { Gestión Alcance } \\
\text { Gestión Tiempo }\end{array}$ \\
\hline Riesgo tecnológico & $\begin{array}{l}\text { Control y mantenimientos preventivos } \\
\text { Investigación y desarrollo tecnológico } \\
\text { Realizar reuniones periódicas, para analizar el desempeño del } \\
\text { sistema }\end{array}$ & $\begin{array}{l}\text { Gestión Alcance } \\
\text { Gestión Tiempo }\end{array}$ \\
\hline Riesgo costos & $\begin{array}{l}\text { Reserva de contingencia del proyecto } \\
\text { Recalculo de los costos estimados, aumentar el presupuesto } \\
\text { formulado } \\
\text { Realizar un buen estudio de proveedores que permita a la empresa } \\
\text { ahorrar costos en el proyecto }\end{array}$ & Gestión Costos \\
\hline
\end{tabular}


Tabla 3 (continuación)

\begin{tabular}{|c|c|c|}
\hline Riesgos financieros & $\begin{array}{l}\text { Uso de derivados financieros para mitigar volatilidad de precios. } \\
\text { Invertir las ganancias derivados del proyecto en mecanismos de uso } \\
\text { eficiente de la energía para mejora continua del sistema. } \\
\text { Administradores de inversiones y cartera } \\
\text { Proteger activos mediante la contratación de seguro }\end{array}$ & Gestión Costos \\
\hline Riesgos externos & $\begin{array}{l}\text { Mecanismos de garantía o de seguro no sólo del riesgo comercial } \\
\text { sino del riesgo político } \\
\text { La protección de las inversiones de largo plazo } \\
\text { Realizar campañas de difusión e integración con las comunidades y } \\
\text { los grupos ambientalistas } \\
\text { Proteger activos mediante la contratación de seguro }\end{array}$ & $\begin{array}{c}\text { Gestión } \\
\text { Alcance } \\
\text { Gestión Costos }\end{array}$ \\
\hline
\end{tabular}

\section{CONCLUSIONES}

De los resultados mostrados, de su análisis y de su discusión, se pueden obtener las siguientes conclusiones sobre la implementación de sistemas de energía solar fotovoltaicos como alternativa para suplir la demanda energética de las operaciones de extracción de oro en Colombia

Toda implementación de sistemas de energía solar fotovoltaicos debe ir acompañada de un plan que identifique y priorice los riesgos a partir de criterios técnicos y económicos. El alcance de este implica desarrollar un proceso en gestión de los riesgos, en la toma de decisiones y el análisis multicriterio. La importancia de este plan radica en que explora nuevas alternativas y las combina en función de hallar respuestas que aporten soluciones a los temas relacionados con la gestión de los riesgos.

La evaluación de los expertos pudo definir que riesgos a nivel tecnológico, operacional, de diseño, económico, de costos, financiero y externo. Una de las limitaciones de la metodología AHP es que no entrega una calificación del riesgo de acuerdo con el nivel de criticidad (alto, medio y bajo); solo pondera y prioriza los riesgos de acuerdo con las comparaciones realizadas por el decisor y define qué fase del proyecto sufre más afectación por la aparición de esos riesgos.

Es importante resaltar que la gestión de los riesgos es un proceso que no debe tomarse a la ligera. Desafortunadamente, en el entorno y la cultura colombiana, donde los resultados importan más que el procedimiento, no se da la importancia debida a este tipo de análisis, dando por hecho que el tratamiento de los riesgos es el mismo para casi todos los casos. Pese a que trabajar con la metodología AHP implica una mayor inversión de tiempo y esfuerzo por los involucrados en el proyecto, ésta tiene como gran ventaja que el análisis de los riesgos se realiza de manera estructurada, coherente y tiene un soporte matemático, haciendo que los resultados adquieran mayor validez.

\section{REFERENCIAS}

Al Garni, H., A. Kassem y otros cuatro autores, Decision Making Approach for Evaluating Renewable Power Generation Sources In Saudi Arabia, Sustainable Energy Technologies and Assessments, 16, 137-150 (2016)

Cardona, O. D., La necesidad de representar de manera holística los conceptos de vulnerabilidad y riesgo "Una crítica y una Revisión Necesaria para la Gestión", Centro de estudios sobre Desastres y Riesgos CEDERI, Universidad de Los Andes, 11 (2001)

De Almeida, A.T., M.H. Alencar y otros dos autores, A Systematic Literature Review of Multicriteria and Multi-objective Models Applied in Risk Management, IMA Journal of Management Mathematics, 28(2), 153-184 (2017)

Del Sol, F. y E. Sauma, Economic Impacts of Installing Solar Power Plants in Northern Chile, Renewable and Sustainable Energy Reviews, 19, 489-498 (2013)

Forbes, D., S. Smith y M. Horner, Tools for Selecting Appropriate Risk Management Techniques in the Built Environment, Construction management and economics, 26 (11), 1241-1250 (2008)

Guerrero Liquet, G., M. García-Cascales y otros dos autores, Risk Management in the Renewable Energy field: Comparative Analysis and Study case in the Dominican Republic, $19^{\text {th }}$ International Congress on Project Management and Engineering, Granada, 15-17th July (2015)

Lin, C., e Y. Shiue, An Application of AHP and Sensitivity Analysis for Measuring the Best Strategy of Reverse Logistics: A Case Study of Photovoltaic Industry Chain, Journal of Testing and Evaluation, 41(3), 386-397 (2013)

Mustafa, M.A. y J.F. Al-Bahar, Project Risk Assessment Using the Analytic Hierarchy Process, IEEE Transactions on Engineering Management, 38(1), 46-52 (1991)

Parrado, C., A. Girard, F. Simon y E. Fuentealba, 2050 LCOE (Levelized Cost of Energy) Projection for a Hybrid PV (Photovoltaic)-CSP (Concentrated Solar Power) Plant in the Atacama Desert, Chile, Energy, 94, 422-430 (2015) 
Prasanta Kumar Dey, Decision Support System for Risk Management: A Case Study. Management Decision, 39(8), 634649 (2001)

PRONACOSE, Sustento Numérico del Software Superdecisions en el Análisis Multicriterio del Tipo AHP/ANP (2014)

Saaty. R. W., Decision Making in Complex Environments. The Analytic Hierarchy Process (AHP) for Decision Making and the Analytic Network Process (ANP) for Decision Making with Dependence and Feedback (2003)

Saaty, T. L., Risk-Its Priority and Probability: The Analytic Hierarchy Process, Risk Analysis, 7(2), 159-172 (1987)

Saaty, T. L., L.G. Vargas, Models, Methods, Concepts \& Applications of the Analytic Hierarchy Process, Vol. 175, Springer Science \& Business Media (2012)

Toskano H.G., El Proceso de Análisis Jerárquico (AHP) como Herramienta para la Toma de Decisiones en la Selección de Proveedores, Tesis, Universidad Nacional Mayor de San Marcos, Facultad de Ciencias Matemáticas, Perú (2009) 